\title{
Quantitative Concept
}

National Cancer Institute

\section{Source}

National Cancer Institute. Quantitative Concept. NCI Thesaurus. Code C70766.

Capable of being estimated or expressed with numeric values. 